Research Article

\title{
Effects of Focal Vibration over Upper Limb Muscles on the Activation of Sensorimotor Cortex Network: An EEG Study
}

\author{
Wei Li, ${ }^{1}$ Chong Li, ${ }^{1}$ Quan Xu, ${ }^{2}$ and Linhong Ji ${ }^{1}{ }^{1}$ \\ ${ }^{1}$ Division of Intelligent and Biomechanical System, State Key Laboratory of Tribology, Department of Mechanical Engineering, \\ Tsinghua University, Haidian, Beijing, China \\ ${ }^{2}$ Department of Rehabilitation Medicine, Beijing Tsinghua Changgung Hospital, School of Clinical Medicine, \\ Tsinghua University, Beijing, China \\ Correspondence should be addressed to Linhong Ji; jilh@tsinghua.edu.cn
}

Received 8 October 2018; Revised 8 April 2019; Accepted 12 May 2019; Published 27 May 2019

Guest Editor: Stuart Cunningham

Copyright ( $\odot 2019$ Wei Li et al. This is an open access article distributed under the Creative Commons Attribution License, which permits unrestricted use, distribution, and reproduction in any medium, provided the original work is properly cited.

\begin{abstract}
Studying the therapeutic effects of focal vibration (FV) in neurorehabilitation is the focus of current research. However, it is still not fully understood how FV on upper limb muscles affects the sensorimotor cortex in healthy subjects. To explore this problem, this experiment was designed and conducted, in which FV was applied to the muscle belly of biceps brachii in the left arm. During the experiment, electroencephalography (EEG) was recorded in the following three phases: before FV, during FV, and two minutes after FV. During FV, a significant lower relative power at C3 and C4 electrodes and a significant higher connection strength between five channel pairs (Cz-FC1, Cz-C3, Cz-CP6, C4-FC6, and FC6-CP2) in the alpha band were observed compared to those before FV. After FV, the relative power at $\mathrm{C} 4$ in the beta band showed a significant increase compared to its value before $\mathrm{FV}$. The changes of the relative power at $\mathrm{C} 4$ in the alpha band had a negative correlation with the relative power of the beta band during FV and with that after FV. The results showed that FV on upper limb muscles could activate the bilateral primary somatosensory cortex and strengthen functional connectivity of the ipsilateral central area $(\mathrm{FC} 1, \mathrm{C} 3$, and $\mathrm{Cz})$ and contralateral central area (CP2, Cz, C4, FC6, and CP6). These results contribute to understanding the effect of FV over upper limb muscles on the brain cortical network.
\end{abstract}

\section{Introduction}

In the past few years, more effort has been paid to studying the effects of focal vibration (FV) at a high frequency $(50 \sim 120 \mathrm{~Hz})$ and with a low amplitude on the rehabilitation of neurological diseases, such as stroke, spinal cord injury, multiple sclerosis, and cerebral palsy [1]. As for patients with stroke, FV can improve various abilities and functionalities, including walking [2], postural sway and gait ability [3], motor performances of reaching movement [4], stability of the proximal arm [5], and reducing spasticity [6, 7]. As for patients with spinal cord injury, FV reduced spasticity [1] and elicited stepping movements [8]. Additionally, research indicated FV can also contribute to the improvement of movement control in patients with multiple sclerosis [9].

The neurophysiological mechanism underlying how FV benefits the recovery of motor function for patients with neurological diseases has been explored mainly in experiments with healthy subjects. At spinal cord level, some studies have shown that FV induced the firing of muscle spindle primary endings (Ia afferent fibers) [10-12]. At the cortical level, studies using transcranial magnetic stimulation (TMS) showed that FV enhanced the excitability of motorcortical representation of vibrated muscle $[6,13]$. Besides, some researchers also have proved that vibrotactile stimulation on the palm or fingers caused the activation of primary motor cortex (M1), primary somatosensory cortex (S1), and secondary somatosensory cortex (S2) using functional magnetic resonance imaging (fMRI) [14-18]. However, how FV applied to other body sites influence the activation of sensorimotor cortex has not been fully studied.

Electroencephalography (EEG) is an electrophysiological method to record the electrical activity of the brain. The rolandic alpha rhythm (sensorimotor "mu" rhythms) 
concentrates mainly in the somatosensory postcentral gyrus, while the rolandic beta rhythm (sensorimotor beta rhythms) mainly originates in the precentral motor cortex $[19,20]$. It was well accepted that event-related desynchronization (ERD) (the decrease of power) over the sensorimotor areas represented the activation of the sensorimotor cortex. On the contrary, event-related synchronization (ERS) (the increase of power) reflected the deactivation of the sensorimotor cortex $[21,22]$. Some EEG-fMRI studies also showed that the decrease of EEG power was related to activation of the sensorimotor cortex [19, 23-25]. As for functional connectivity, it can reflect the level of synchronization between the signals of different scalp regions, as well as the topological and dynamics properties of information flow between different brain areas $[26,27]$. Recently, it has been used as a biomarker to investigate the mechanism of functional recovery in patients with neurological diseases [28-31].

In this study, we aimed to study the effect of FV applied over left biceps brachii on the sensorimotor cortex during FV and after FV. We recorded the EEG activity before FV, during FV, and $2 \mathrm{~min}$ after FV. Then, we analyzed the changes of relative power at $\mathrm{C} 3$ and $\mathrm{C} 4$ and functional connectivity of the central region (FC5, FC1, FC2, FC6, C3, $\mathrm{Cz}, \mathrm{C} 4, \mathrm{CP} 5, \mathrm{CP} 1, \mathrm{CP} 2$, and $\mathrm{CP} 6)$ in the alpha $(8-12 \mathrm{~Hz})$ and beta $(12.5-30 \mathrm{~Hz})$ band.

\section{Materials and Methods}

2.1. Participants. Twenty male right-handed healthy subjects with a mean age of 26 years ( \pm 0.6 years) were recruited in Tsinghua University in this study. All subjects were informed about the procedure of the experiment. All the subjects gave written consent prior to the experiment. The study was approved by the institutional ethics committee.

2.2. Experimental Setup. All the participants were informed to have a good rest one day before the experiment in order to minimize drowsiness. During the experiment, each subject was seated in a comfortable chair with both arms on the armrest and their hand supinated so that their upper limbs were relaxed. FV with frequency at $75 \mathrm{~Hz}$ and amplitude at $1.2 \mathrm{~mm}$ was produced by a mechanical vibration device with a vibration head (YS-889, Jialemei Health Care Co., Ltd., Taiwan, China), as shown in Figure 1. The mechanical vibration device was operated at a power frequency of $50 \mathrm{~Hz}$. FV was applied perpendicularly over the muscle belly of biceps brachii in the left arm lasting for three minutes. During the EEG recording, subjects were asked to minimize head movement, eye movement, body movement, and chewing and were required to wear earplugs to reduce external noise and vibration noise. Based on the recommendation to use eyes-closed resting EEG as a baseline for tasks without visual stimuli [32], all the subjects were asked to keep their eyes closed during this experiment. Specifically, EEG was collected in the following three phases: (1) before FV (baseline: before-FV), the resting state EEG was recorded with the eyes closed for $4 \mathrm{~min}$; (2) during FV (during-FV),

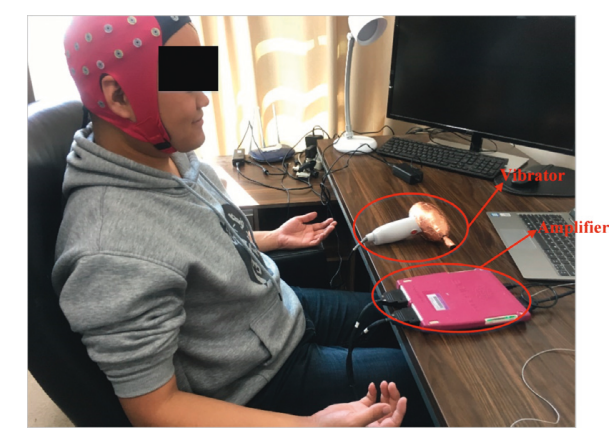

FIGURE 1: Illustration of the experimental setup.

EEG were recorded with the eyes closed for $3 \mathrm{~min}$ of vibration; and (3) after FV (after-FV), EEG were recorded with the eyes closed for $3 \mathrm{~min}$, starting at 2 minutes after the termination of $\mathrm{FV}$.

2.3. EEG Recordings. EEG signals were recorded with ANT hardware and software (B.V., Enschede, the Netherlands) from $32 \mathrm{Ag} / \mathrm{AgCl}$ electrodes mounted in a commercial WaveGuard EEG Cap (Eemagine Medical Imaging Solutions GmbH, ANT Advanced Neuro Technology) and positioned over the whole scalp according to the international 10-20 system, as well as two electrodes on the left and right mastoids. A ground reference electrode was located between the $\mathrm{Fpz}$ and $\mathrm{Fz}$ electrode, and the reference electrode was located at the $\mathrm{Cpz}$ electrode. The sampling rate was set at $1000 \mathrm{~Hz}$. Electrode impedances were kept below $5 \mathrm{k} \Omega$.

\subsection{EEG Signal Analysis}

2.4.1. Signal Preprocessing. The EEG signals were preprocessed in EEGLAB 14 (EEGLAB toolbox, Swartz Center for Computational Neurosciences, La Jolla, CA; http://www. sccn.ucsd.edu/eeglab). Data were divided into segments of $2 \mathrm{~s}$. Artifacts were visually detected. The data were referenced to the common average reference. The power line noise $50 \mathrm{~Hz}$ was removed. A band-pass filter was set between $0.5 \mathrm{~Hz}$ and $50 \mathrm{~Hz}$. The Welch method (pWelch algorithm, an overlapping 1-second hanning window, no phase shift) was applied to compute the power spectral density of each epoch ( 2 second duration, 2000 data points) using a $0.5 \mathrm{~Hz}$ frequency resolution, and all the epochs were then averaged.

2.4.2. Relative Power Analysis. In this study, relative power was estimated in the two frequency bands: alpha $(8-12 \mathrm{~Hz})$ and beta $(12.5-30 \mathrm{~Hz})$. The relative power $\mathrm{RP}($.$) was cal-$ culated as follows:

$$
\operatorname{RP}\left(f_{1}, f_{2}\right)=\frac{\int_{f_{1}}^{f_{2}} \operatorname{PSD}\left(f_{1}, f_{2}\right) d f}{\int_{f_{1}}^{f_{2}} \operatorname{PSD}(0.5,50) d f},
$$

where $f_{1}$ and $f_{2}$ indicate the low and high frequency of the specified frequency band, respectively. $\operatorname{PSD}($.$) indicates the$ power spectral density. 
Alpha and beta motor-related power desynchronizations (MRPD) at C3 and C4 electrodes were used to indicate the activation of primary sensorimotor cortex [33]. MRPP was calculated as follows:

$$
\begin{aligned}
\mathrm{MRPD}_{\text {during-FV }} & =\frac{\mathrm{RP}_{\text {during-FV }}-\mathrm{RP}_{\text {baseline }}}{\mathrm{RP}_{\text {baseline }}}, \\
\mathrm{MRPD}_{\text {after-FV }} & =\frac{\mathrm{RP}_{\text {after-FV }}-\mathrm{RP}_{\text {baseline }}}{\mathrm{RP}_{\text {baseline }}},
\end{aligned}
$$

where $\mathrm{RP}_{\text {baseline, }} \mathrm{RP}_{\text {during-FV }}$, and $\mathrm{RP} \mathrm{P}_{\text {after-FV }}$ indicate the relative power before $\mathrm{FV}$, during $\mathrm{FV}$, and after $\mathrm{FV}$, respectively. The negative or positive values reflected alpha (beta) movement-related power desynchronization or synchronization, respectively.

2.4.3. Functional Connectivity Analysis. In this study, functional connectivity was estimated using imaginary coherence, which reduced overestimation biases that exist in many other measures, such as phase locking value, absolute coherence, and synchronization likelihood [34-36]. Due to the common reference, cross-talk, and volume conduction, these measures generated spurious interactions with no time lag. Imaginary coherence was expressed as the imaginary part of coherency $C_{i j}(f)$, which was defined as the normalized cross-spectrum:

$$
C_{i j}(f)=\frac{S_{i j}(f)}{\left(S_{i i}(f) S_{j j}(f)\right)^{1 / 2}},
$$

where $S_{i j}(f) \equiv\left\langle x_{i}(f) x_{j}^{*}(f)\right\rangle$ is the cross-spectrum. $x_{i}(f)$ and $x_{j}(f)$ indicate the complex Fourier transforms of the time series $\hat{x}_{i}(t)$ and $\hat{y}_{j}(t)$ of the channel $i$ and $j$, respectively. \langle\rangle indicates expectation value and $*$ indicates complex conjugation. Expectation value was estimated as an average over all the segments.

In this study, the functional connectivity of the central region (FC5, FC1, FC2, FC6, C3, Cz, C4, CP5, CP1, CP2, and CP6) was calculated.

2.5. Statistical Analysis. The statistical analysis was performed by SPSS Statistics 20. In the analysis of relative power, two-way repeated measures analysis of variance (ANOVA) was used. Before two-way repeated ANOVA was performed, the Shapiro-Wilk test was used to determine if all the data sets of relative power were well-modeled by a normal distribution. The ANOVA factors included the main factor condition (before FV, during FV, and after FV) and the main factor electrode (C3 and C4). If the main factor condition or electrode showed a significance, a pairedsample $t$-test was then performed. In the statistical analysis of functional connectivity, there were a large number of tested channel pairs $\left(C_{11}^{2}=55\right)$. To make sure the probability of one or more null hypotheses incorrectly rejected, false discovery rate (FDR) correction was performed. The Type I error was set to 0.05 . Pearson's correlation analysis was performed between alpha MRPD and beta MRPD during and after FV.

\section{Results}

3.1. Relative Power Analysis. In the alpha band, the Shapiro-Wilk test showed relative power at C3 $(p=0.991$, 0.867 , and 0.438) and C4 $(p=0.126,0.097$, and 0.156) during three phases, before FV, during-FV, and after-FV was normally distributed. Two-way repeated-measures ANOVA showed the interaction electrode $\times$ condition $(F(2,38)=1.137 ; p=0.331)$ and the main factor electrode $(F(1,19)=2.480 ; p=0.132)$ were not significant, while the main factor condition $(F(2,38)=4.718 ; p=0.015)$ was significant. A paired-sample $t$-test for the relative power at $\mathrm{C} 3$ and $\mathrm{C} 4$ showed a significant decrease during FV (one tailed: $p=0.0095$ and 0.0075) and after FV (one tailed: $p=0.0145$ and 0.037 ) compared to before-FV. After the Bonferroni correction, the relative power of C3 and C4 during FV showed a significant decrease (one tailed: $p=0.019$ and 0.015), whereas the relative power of C3 after FV showed a significant decrease (one tailed: $p=0.029$ ) (Figure 2).

In the beta band, the Shapiro-Wilk test showed relative power at C3 $(p=0.192,0.709$, and 0.510$)$ and C4 $(p=$ $0.348,0.055$, and 0.923 ) during three phases, before-FV, during-FV, and after-FV, was normally distributed. Twoway repeated-measures ANOVA revealed that the interaction electrode $\times$ condition $(F(2,38)=0.323 ; p=0.726)$ and the main factor electrode $(F(1,19)=2.135 ; p=0.160)$ were not significant, while the main factor condition $(F(2,38)=4.818 ; p=0.014)$ was significant. The pairedsample $t$-test for the relative power at C3 and C4 showed a significant increase after FV compared to before-FV (one tailed: $p=0.033$ and 0.017$)$. After the Bonferroni correction, the relative power of $\mathrm{C} 4$ after FV showed a significant increase compared to before-FV $(p=0.034)$, as shown in Figure 2 .

3.2. Pearson's Correlation Analysis. The values of MRPD at $\mathrm{C} 3$ and $\mathrm{C} 4$ in the alpha and beta bands are shown in Figure 3. A significant negative correlation was found between MRPD at $\mathrm{C} 4$ in the alpha band and one in the beta band during FV $(p=0.03$, Pearson's $r=-0.43)$, as well as after FV ( $p=0.043$, Pearson's $r=-0.39$ ) (Figure 3). No significant correlation was found between MRPD at C3.

3.3. Functional Connectivity Analysis. After FDR correction tests, the connection strength of $\mathrm{Cz}-\mathrm{CP} 6, \mathrm{Cz}-\mathrm{FC} 1, \mathrm{Cz}-\mathrm{C} 3$, C4-FC6, and FC6-CP2 in the central region showed a significant increase in the alpha band during FV, which is shown in Figure 4. The $p$ values of connection strength of five channel pairs were 0.0093, 0.0259, 0.0261, 0.0047, and 0.0351 , respectively.

\section{Discussion}

In order to investigate the effect of FV applied over upper limb muscles on sensorimotor cortex during FV and after FV, the experiment was conducted in which FV was delivered to the muscle belly of biceps brachii in the left arm and EEG was monitored in three phases, before FV, during 


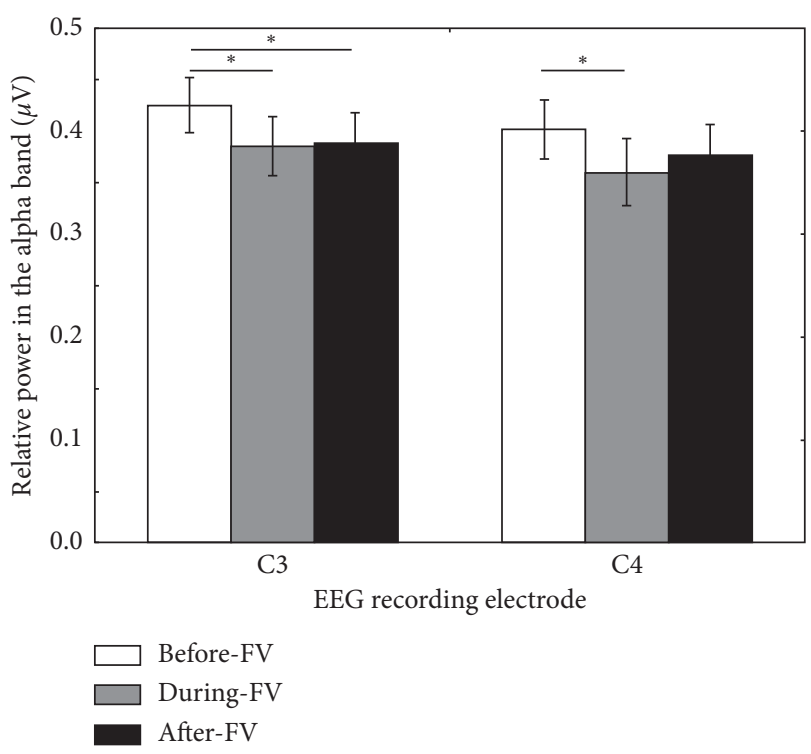

(a)

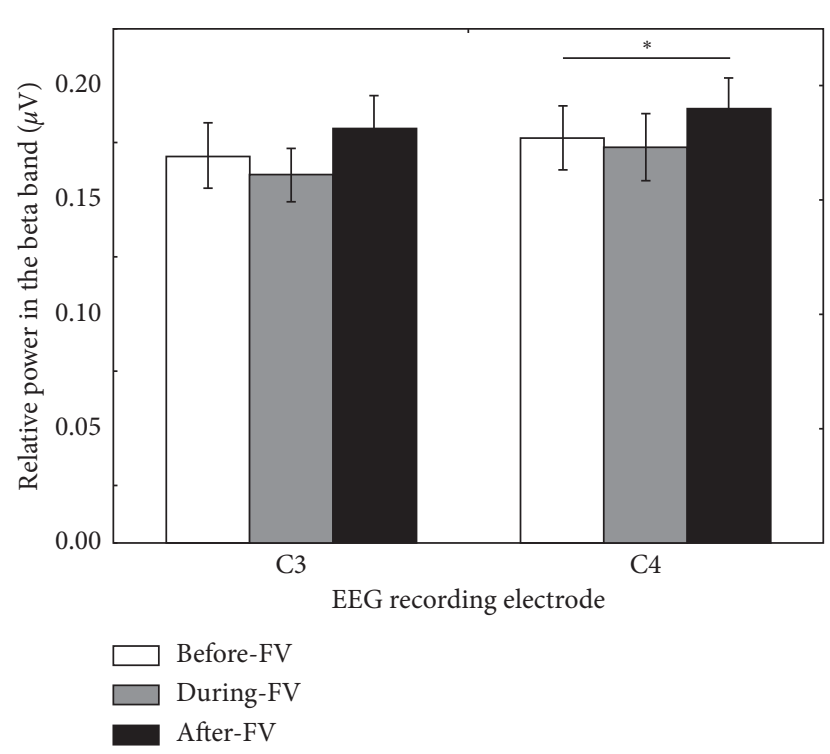

(b)

FIgURE 2: Relative power of the alpha band (a) and beta band (b) at C3 and C4 before FV (white), during FV (grey), and after FV (black). Note: mean values of relative power with SEM are present in charts; asterisks indicate significant differences $(p<0.05)$.

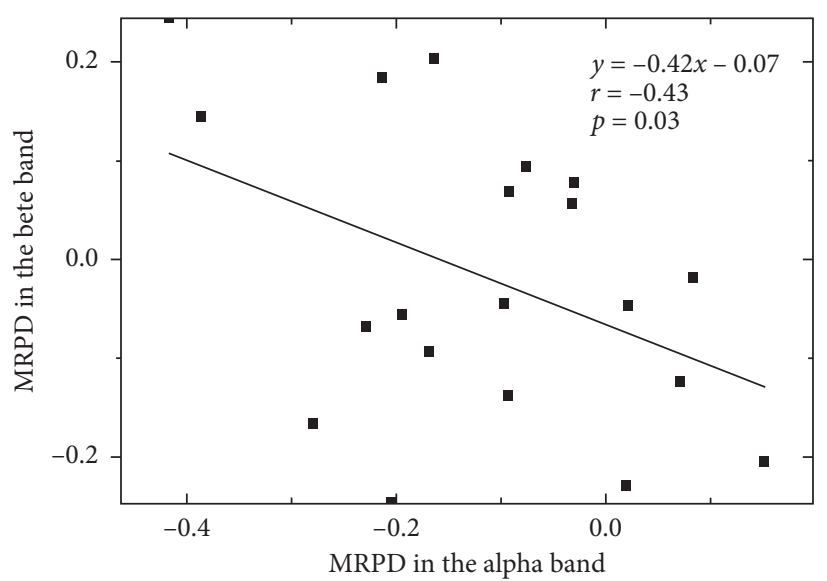

(a)

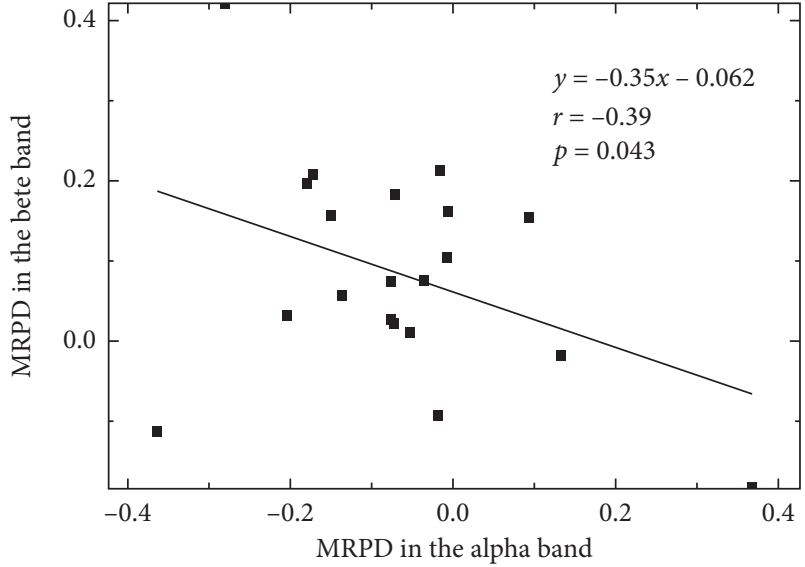

(b)

FIgURE 3: Pearson's correlation between alpha MRPD and beta MRPD during FV (a) and after FV (b) at C4. Solid squares indicate each subject's MRPD.

$\mathrm{FV}$, and after FV. The results showed that FV on upper limb muscles could activate bilateral S1 and strengthen connection strength of the central region, including $\mathrm{Cz}-\mathrm{FC} 1, \mathrm{Cz}-\mathrm{C} 3$, $\mathrm{Cz}-\mathrm{CP} 6, \mathrm{C} 4-\mathrm{FC} 6$, and FC6-CP2. The effect could not be maintained two minutes after FV. We also find that the changes of relative power at $\mathrm{C} 4$ in the alpha band have a negative correlation with the ones in the beta band during FV and after FV.

4.1. Before FV and during FV. In the present study, the results show that the application of FV over the muscle belly of biceps brachii in the left arm can activate contralateral S1. The "mu" rhythms originate mainly in the somatosensory postcentral gyrus $[19,20]$. Moreover, the previous studies found that desynchronized power in the alpha band had a positive correlation with the activation of S1 [20,23-25]. The present result was in line with the previous studies showing that vibrotactile at the palm or finger could activate contralateral S1 using fMRI $[14,16,18]$. It also meant that FV, which was similar to motor preparation, motor execution, motor imagery, and somatosensory stimuli, induced alphaERD pattern $[22,37,38]$. There was a consensus that muscle spindle and cutaneous mechanoreceptors, like Merkel afferents, Meissner afferents, and Pacinian afferents, responded to $\mathrm{FV}$ at the frequency of $75 \mathrm{~Hz}$ applied over muscle belly [39]. Based on this, it could be inferred that the activation of S1 could result from two pathways. One way is that the proprioceptive information from muscle spindle travels along the upper body proprioceptive pathway, which 


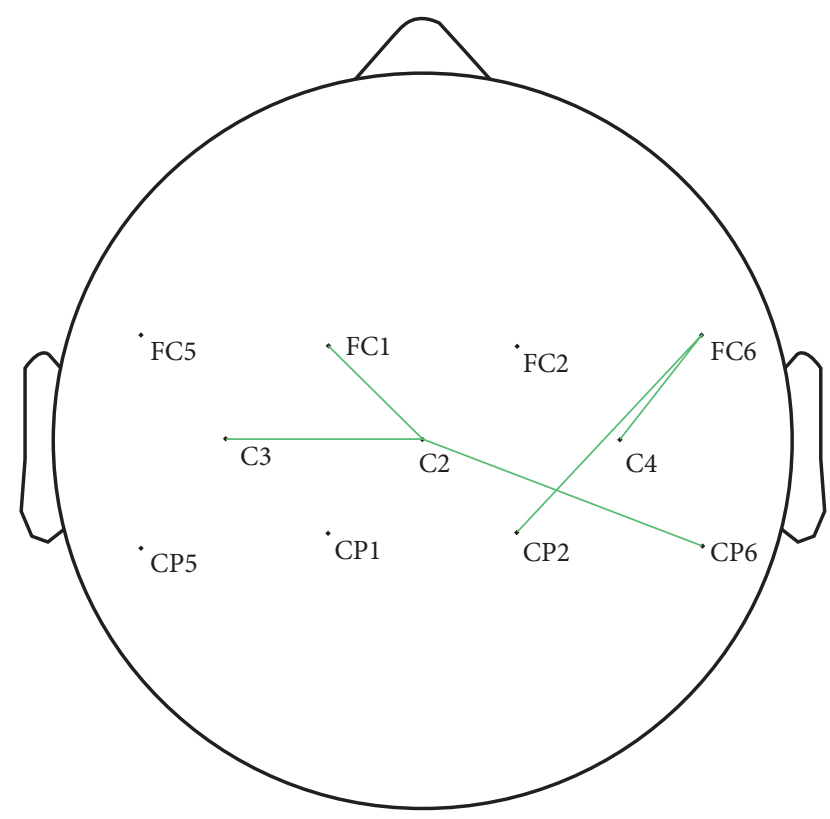

FIGURE 4: Connection strength of different channel pairs with significant differences during FV in the alpha band after FDR correction.

decussates in the caudal medulla through the posterior column-medial lemniscus pathway, finally to reach S1 through the ventral posterior lateral nucleus of the thalamus. The other pathway is that this cutaneous mechanoreceptor information conveys by a separate set of first-order neurons located in the trigeminal ganglion, then ascends to the ventral posterior medial nucleus of the thalamus through the neurons given off by the trigeminal brainstem nuclei, and finally reaches S1 [39]. Besides, the present result shows the occurrence of the activation of ipsilateral S1 following FV, which could originate from the input of about $10 \%$ uncrossed corticospinal tracts [40]. The activation of ipsilateral S1 following FV is also found in several studies $[14,15]$.

In the present study, the connection strength of $\mathrm{Cz}-\mathrm{FC} 1$, Cz-C3, Cz-CP6, C4-FC6, and FC6-CP2 can be strengthened following FV. Some studies showed that C3 and C4 could project close to postcentral gyrus whilst $\mathrm{Cz}$ and $\mathrm{FC} 1$ could project close to precentral gyrus $[35,41,42]$. It seemed that the connection strength between ipsilateral postcentral gyrus and precentral gyrus is strengthened, which is similar to earlier findings indicating that functional connectivity of the ipsilateral sensorimotor area during real movements was strengthened [43].

4.2. Before FV and after FV. After FV, the present study shows that the relative power at $\mathrm{C} 4$ in the beta band has a statistically significant increase, which indicates the rebound of beta power after FV. It has been generally accepted that beta rebound coincided with reduced excitability of motor cortex neurons, which is similar to the phenomenon after active movement, passive movement, motor imagery, and somatosensory stimulation [21, 44-46]. Based on "functional inhibition" hypothesis, a desynchronized alpha band following the occurrence of a synchronized power in the beta band could reflect a mechanism of functional inhibition of the motor cortex by somatosensory processing [47]. It could be inferred that the activation of somatosensory cortex induced by FV could inhibit the excitability of motor cortex after FV. In the present study, the occurrence of alpha MRPD and beta MRPD at C4 shows a significant negative correlation between during-FV and after-FV. It is in line with the previous result showing that a stronger mu rhythm ERD appeared with an enhanced beta ERS with foot movement [22]. Besides, no significant reduction of relative power at $\mathrm{C} 4$ in the alpha band showed that the excitability of S1 was not maintained about two minutes after FV.

The differences between our findings and the current literature are that M1 is not activated and the duration of the excitability of sensorimotor areas induced by FV is shorter in this study. In the previous fMRI studies, FV applied at the hand palm or finger could activate M1 [14, 15, 18]. On the one hand, the number of muscle spindles per gram of muscle tissue at other sites of limb muscles was lower than at the hand palm $(130 / g)[14,48]$. The proprioceptive input is too weak to induce the activation of M1. One the other hand, it might be ascribed to the difference in the amplitude of FV. One study showed that vibration stimulus with low amplitude $(0.4 \mathrm{~mm})$ activated sensory and motor cortex more strongly than high amplitude $(1.6 \mathrm{~mm})$ [17]. In addition, the duration of effect could be related to the lasting time of FV. There were several studies using TMS showing that the excitability of sensorimotor cortex was not enhanced shortly after short-lasting FV [49-51], whilst the excitability of sensorimotor cortex lasted for longer time after long-lasting vibration stimulation [52]. Recently, one study also showed that FV could increase the excitability of S1-M1 immediately after FV, but the time of FV lasted for about thirty minutes [33].

Notably, our results show that FV could activate S1 and strengthen the functional connectivity of the sensorimotor system. The activation of S1 could play an important role in the recovery of motor function in patients with neurological diseases. On the one hand, somatosensory input from FV to the motor cortex, via corticocortical connections with S1, plays a critical role in motor relearning after hemiparetic stroke $[53,54]$. On the other hand, FV, as one of the most effective modulators of cortical structure and function, could modify synaptic efficacy and transmission and as a consequence causes a cortical reorganization of the somatosensory representational maps. The reorganization of function and structure could contribute to the recovery of limb motor function. In the present study, the results using EEG are similar to ones using fMRI. It could be an appropriate way for EEG to further investigate the underlying neurophysiological mechanisms of $\mathrm{FV}$ on rehabilitation of motor function.

One important limitation of this study is that this is a pilot study, but not a randomized controlled trial. It cannot be precluded that part of the effects occurred could be due to the experimental procedure and not due to vibration stimulation. Nevertheless, our findings indicate that FV 
could activate the sensorimotor cortex and strengthen the connection strength between central regions by a comparison of before-FV with during-FV. It can be feasible to explore the effect of FV on sensorimotor cortex using EEG for later use on clinical research. In a future clinical experiment, a randomized controlled trial will be designed to explore the mechanism of $\mathrm{FV}$ in motor rehabilitation using EEG.

\section{Conclusion}

Our study shows that FV on upper limb muscles could activate the activity of primary somatosensory cortex and strengthen connection strength of the central region. Based on our present results, we will investigate the effect of FV on the activation of sensorimotor cortex for stroke patients, to further explore the underlying neurophysiological mechanisms of FV on rehabilitation of motor function in the clinical experiment.

\section{Data Availability}

The EEG data used to support the findings of this study are available from the corresponding author upon request.

\section{Conflicts of Interest}

The authors declare that they have no financial or personal conflicts of interest.

\section{Acknowledgments}

This work was supported by the National Natural Science Foundation of China (Grant nos. U1613207 and 51805288). The authors are grateful to the National Natural Science Foundation of China and the subjects who participated in the study.

\section{References}

[1] N. Murillo, J. Valls-Sole, J. Vidal, E. Opisso, J. Medina, and H. Kumru, "Focal vibration in neurorehabilitation," European Journal of Physical and Rehabilitation Medicine, vol. 50, no. 2, pp. 231-242, 2014.

[2] M. Paoloni, M. Mangone, P. Scettri, R. Procaccianti, A. Cometa, and V. Santilli, "Segmental muscle vibration improves walking in chronic stroke patients with foot drop: a randomized controlled trial," Neurorehabilitation and Neural Repair, vol. 24, no. 3, pp. 254-262, 2010.

[3] S.-W. Lee, K.-H. Cho, and W.-H. Lee, "Effect of a local vibration stimulus training programme on postural sway and gait in chronic stroke patients: a randomized controlled trial," Clinical Rehabilitation, vol. 27, no. 10, pp. 921-931, 2013.

[4] E. Tavernese, M. Paoloni, M. Mangone et al., "Segmental muscle vibration improves reaching movement in patients with chronic stroke: a randomized controlled trial," Neurorehabilitation and Neural Repair, vol. 32, no. 3, pp. 591-599, 2013.

[5] M. O. Conrad, R. A. Scheidt, and B. D. Schmit, "Effects of wrist tendon vibration on targeted upper-arm movements in poststroke hemiparesis," Neurorehabilitation and Neural Repair, vol. 25, no. 1, pp. 61-70, 2011.
[6] B. Marconi, G. M. Filippi, G. Koch et al., "Long-term effects on cortical excitability and motor recovery induced by repeated muscle vibration in chronic stroke patients," Neurorehabilitation and Neural Repair, vol. 25, no. 1, pp. 48-60, 2011.

[7] R. S. Calabrò, A. Naro, M. Russo et al., "Is two better than one? Muscle vibration plus robotic rehabilitation to improve upper limb spasticity and function: a pilot randomized controlled trial," PLoS One, vol. 12, no. 10, Article ID e0185936, 2017.

[8] E. Field-Fote, L. L. Ness, and M. Ionno, "Vibration elicits involuntary, step-like behavior in individuals with spinal cord injury," Neurorehabilitation and Neural Repair, vol. 26, no. 7, pp. 861-869, 2012.

[9] P. Feys, W. F. Helsen, S. Verschueren et al., "Online movement control in multiple sclerosis patients with tremor: effects of tendon vibration," Movement Disorders, vol. 21, no. 8, pp. 1148-1153, 2006.

[10] D. Burke, K. E. Hagbarth, L. Löfstedt, and B. G. Wallin, "The responses of human muscle spindle endings to vibration of non-contracting muscles," Journal of Physiology, vol. 261, no. 3, pp. 673-693, 1976.

[11] J. P. Roll and J. P. Vedel, "Kinaesthetic role of muscle afferents in man, studied by tendon vibration and microneurography," Experimental Brain Research, vol. 47, no. 2, pp. 177-190, 1982.

[12] J. P. Roll, J. P. Vedel, and E. Ribot, "Alteration of proprioceptive messages induced by tendon vibration in man: a microneurographic study," Experimental Brain Research, vol. 76, no. 1, pp. 213-222, 1989.

[13] K. Rosenkranz and J. C. Rothwell, "Differential effect of muscle vibration on intracortical inhibitory circuits in humans," Journal of Physiology, vol. 551, no. 2, pp. 649-660, 2003.

[14] S. M. Golaszewski, C. M. Siedentopf, E. Baldauf et al., "Functional magnetic resonance imaging of the human sensorimotor cortex using a novel vibrotactile Stimulator," NeuroImage, vol. 17, no. 1, pp. 421-430, 2002.

[15] S. M. Golaszewski, C. M. Siedentopf, F. Koppelstaetter et al., "Human brain structures related to plantar vibrotactile stimulation: a functional magnetic resonance imaging study," NeuroImage, vol. 29, no. 3, pp. 923-929, 2006.

[16] Y. L. Hegner, R. Saur, R. Veit et al., "BOLD adaptation in vibrotactile stimulation: neuronal networks involved in frequency discrimination," Journal of Neurophysiology, vol. 97, no. 1, pp. 264-271, 2007.

[17] C. M. Siedentopf, K. Heubach, A. Ischebeck et al., "Variability of BOLD response evoked by foot vibrotactile stimulation: influence of vibration amplitude and stimulus waveform," NeuroImage, vol. 41, no. 2, pp. 504-510, 2008.

[18] Y. G. Chung, J. Kim, S. W. Han et al., "Frequency-dependent patterns of somatosensory cortical responses to vibrotactile stimulation in humans: a fMRI study," Brain Research, vol. 1504, pp. 47-57, 2013.

[19] A. Schnitzler, J. Gross, and L. Timmermann, "Synchronised oscillations of the human sensorimotor cortex," Acta Neurobiologiae Experimentalis, vol. 60, no. 2, pp. 271-287, 2000.

[20] P. Ritter, M. Moosmann, and A. Villringer, "Rolandic alpha and beta EEG rhythms' strengths are inversely related to fMRI-BOLD signal in primary somatosensory and motor cortex," Human Brain Mapping, vol. 30, no. 4, pp. 1168-1187, 2009.

[21] G. Pfurtscheller, "Functional brain imaging based on ERD/ ERS," Vision Research, vol. 41, no. 10-11, pp. 1257-1260, 2001.

[22] C. Neuper, M. Wörtz, and G. Pfurtscheller, "ERD/ERS patterns reflecting sensorimotor activation and deactivation," Progress in Brain Research, vol. 159, pp. 211-222, 2006. 
[23] H. Yuan, T. Liu, R. Szarkowski, C. Rios, J. Ashe, and B. He, "Negative covariation between task-related responses in alpha/beta-band activity and BOLD in human sensorimotor cortex: an EEG and fMRI study of motor imagery and movements," NeuroImage, vol. 49, no. 3, pp. 2596-2606, 2010.

[24] E. Formaggio, S. F. Storti, R. Cerini, A. Fiaschi, and P. Manganotti, "Brain oscillatory activity during motor imagery in EEG-fMRI coregistration," Magnetic Resonance Imaging, vol. 28, no. 10, pp. 1403-1412, 2010.

[25] C. Zich, S. Debener, C. Kranczioch, M. G. Bleichner, I. Gutberlet, and M. De Vos, "Real-time EEG feedback during simultaneous EEG-fMRI identifies the cortical signature of motor imagery," NeuroImage, vol. 114, pp. 438-447, 2015.

[26] D. S. Bassett, A. Meyer-Lindenberg, S. Achard, T. Duke, and E. Bullmore, "Adaptive reconfiguration of fractal small-world human brain functional networks," Proceedings of the $\mathrm{Na}$ tional Academy of Sciences, vol. 103, no. 51, pp. 19518-19523, 2006.

[27] E. Bullmore and O. Sporns, "Erratum: complex brain networks: graph theoretical analysis of structural and functional systems," Nature Reviews Neuroscience, vol. 10, no. 4, p. 312, 2009.

[28] C. Grefkes and G. R. Fink, "Reorganization of cerebral networks after stroke: new insights from neuroimaging with connectivity approaches," Brain, vol. 134, no. 5, pp. 1264-1276, 2011.

[29] C. R. Gillebert and D. Mantini, "Functional connectivity in the normal and injured brain," Neuroscientist, vol. 19, no. 5, pp. 509-522, 2013.

[30] G. Silasi and T. H. Murphy, "Stroke and the connectome: how connectivity guides therapeutic intervention," Neuron, vol. 83, no. 6, pp. 1354-1368, 2014.

[31] J. Wu, E. B. Quinlan, L. Dodakian et al., "Connectivity measures are robust biomarkers of cortical function and plasticity after stroke," Brain, vol. 138, no. 8, pp. 2359-2369, 2015.

[32] R. J. Barry, A. R. Clarke, S. J. Johnstone, and C. R. Brown, "EEG differences in children between eyes-closed and eyesopen resting conditions," Clinical Neurophysiology, vol. 120, no. 10 , pp. 1806-1811, 2009.

[33] S. Lopez, F. Bini, C. Del Percio et al., "Electroencephalographic sensorimotor rhythms are modulated in the acute phase following focal vibration in healthy subjects," Neuroscience, vol. 352, pp. 236-248, 2017.

[34] G. Nolte, O. Bai, L. Wheaton, Z. Mari, S. Vorbach, and M. Hallett, "Identifying true brain interaction from EEG data using the imaginary part of coherency," Clinical Neurophysiology, vol. 115, no. 10, pp. 2292-2307, 2004.

[35] A. G. Guggisberg, S. M. Honma, A. M. Findlay et al., "Mapping functional connectivity in patients with brain lesions," Annals of Neurology, vol. 63, no. 2, pp. 193-203, 2008.

[36] F. Freyer, M. Reinacher, G. Nolte, H. R. Dinse, and P. Ritter, "Repetitive tactile stimulation changes resting-state functional connectivity-implications for treatment of sensorimotor decline," Frontiers in Human Neuroscience, vol. 6, p. 144, 2012.

[37] G. Pfurtscheller and F. H. Lopes da Silva, "Event-related EEG/ MEG synchronization and desynchronization: basic principles," Clinical Neurophysiology, vol. 110, no. 11, pp. 18421857, 1999.

[38] C. Babiloni, C. Del Percio, L. Arendt-Nielsen et al., "Cortical EEG alpha rhythms reflect task-specific somatosensory and motor interactions in humans," Clinical Neurophysiology, vol. 125, no. 10, pp. 1936-1945, 2014.

[39] D. Purves, G. J. Augustine, D. Fitzpatrick et al., Neuroscience, Sinauer Associates, Sunderland, MA, USA, 2012.
[40] E. Jankowska and S. A. Edgley, "How can corticospinal tract neurons contribute to ipsilateral movements? A question with implications for recovery of motor functions," Neuroscientist, vol. 12, no. 1, pp. 67-79, 2006.

[41] M. Okamoto, H. Dan, K. Sakamoto et al., "Three-dimensional probabilistic anatomical cranio-cerebral correlation via the international 10-20 system oriented for transcranial functional brain mapping," NeuroImage, vol. 21, no. 1, pp. 99-111, 2004.

[42] L. Koessler, L. Maillard, A. Benhadid et al., "Automated cortical projection of EEG sensors: anatomical correlation via the international 10-10 system," Neuroimage, vol. 46, no. 1, pp. 64-72, 2009.

[43] H. Sugata, M. Hirata, T. Yanagisawa et al., "Alpha band functional connectivity corzrelates with the performance of brain-machine interfaces to decode real and imagined movements," Frontiers in Human Neuroscience, vol. 8, p. 620, 2014.

[44] L. M. Parkes, M. C. M. Bastiaansen, and D. G. Norris, "Combining EEG and fMRI to investigate the post-movement beta rebound," NeuroImage, vol. 29, no. 3, pp. 685-696, 2006.

[45] W. Gaetz, J. C. Edgar, D. J. Wang, and T. P. L. Roberts, "Relating MEG measured motor cortical oscillations to resting $\gamma$-Aminobutyric acid (GABA) concentration," NeuroImage, vol. 55, no. 2, pp. 616-621, 2011.

[46] B. E. Kilavik, M. Zaepffel, A. Brovelli, W. A. MacKay, and A. Riehle, "The ups and downs of beta oscillations in sensorimotor cortex," Experimental Neurology, vol. 245, pp. 1526, 2013.

[47] M. Zaepffel, R. Trachel, B. E. Kilavik, and T. Brochier, "Modulations of EEG beta power during planning and execution of grasping movements," PLoS One, vol. 8, no. 3, Article ID e60060, 2013.

[48] S. M. Golaszewski, F. Zschiegner, C. M. Siedentopf et al., "A new pneumatic vibrator for functional magnetic resonance imaging of the human sensorimotor cortex," Neuroscience Letters, vol. 324, no. 2, pp. 125-128, 2002.

[49] S. Siggelkow, A. Kossev, M. Schubert, H.-H. Kappels, W. Wolf, and R. Dengler, "Modulation of motor evoked potentials by muscle vibration: the role of vibration frequency," Muscle \& Nerve, vol. 22, no. 11, pp. 1544-1548, 1999.

[50] A. Kossev, S. Siggelkow, H.-H. Kapels, R. Dengler, and J. D. Rollnik, "Crossed effects of muscle vibration on motorevoked potentials," Clinical Neurophysiology, vol. 112, no. 3, pp. 453-456, 2001.

[51] M. Steyvers, O. Levin, S. M. Verschueren, and S. P. Swinnen, "Frequency-dependent effects of muscle tendon vibration on corticospinal excitability: a TMS study," Experimental Brain Research, vol. 151, no. 1, pp. 9-14, 2003.

[52] M. Christova, D. Rafolt, S. Golaszewski, and E. Gallasch, "Outlasting corticomotor excitability changes induced by $25 \mathrm{~Hz}$ whole-hand mechanical stimulation," European Journal of Applied Physiology, vol. 111, no. 12, pp. 3051-3059, 2011.

[53] S. Dechaumont-Palacin, P. Marque, X. De Boissezon et al., "Neural correlates of proprioceptive integration in the contralesional hemisphere of very impaired patients shortly after a subcortical stroke: an FMRI study," Neurorehabilitation and Neural Repair, vol. 22, no. 2, pp. 154-165, 2008.

[54] J. D. Schaechter, C. A. M. M. van Oers, B. N. Groisser et al., "Increase in sensorimotor cortex response to somatosensory stimulation over subacute poststroke period correlates with motor recovery in hemiparetic patients," Neurorehabilitation and Neural Repair, vol. 26, no. 4, pp. 325-334, 2012. 


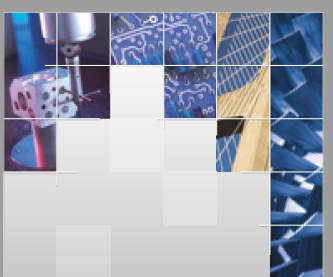

\section{Enfincering}
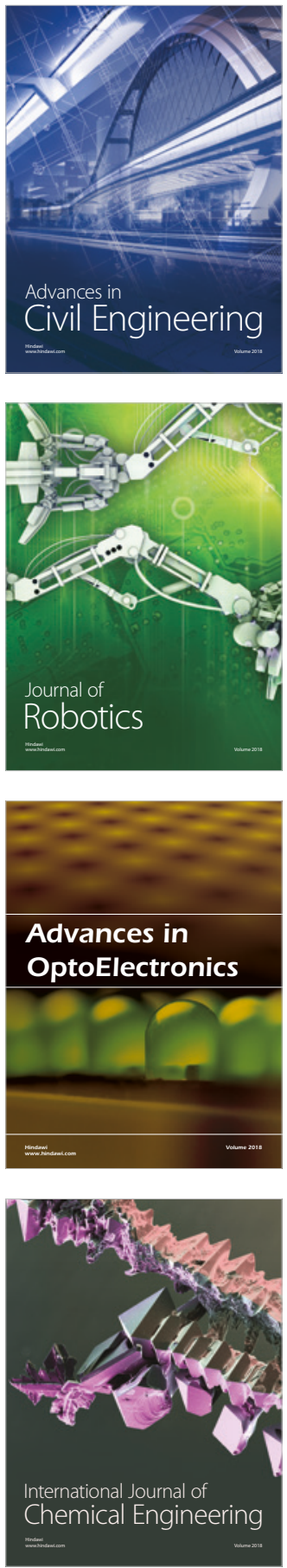

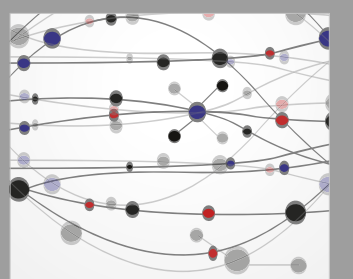

\section{Rotating \\ Machinery}

The Scientific World Journal

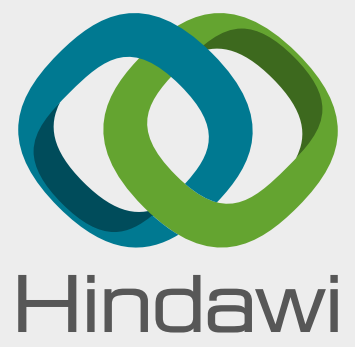

Submit your manuscripts at

www.hindawi.com
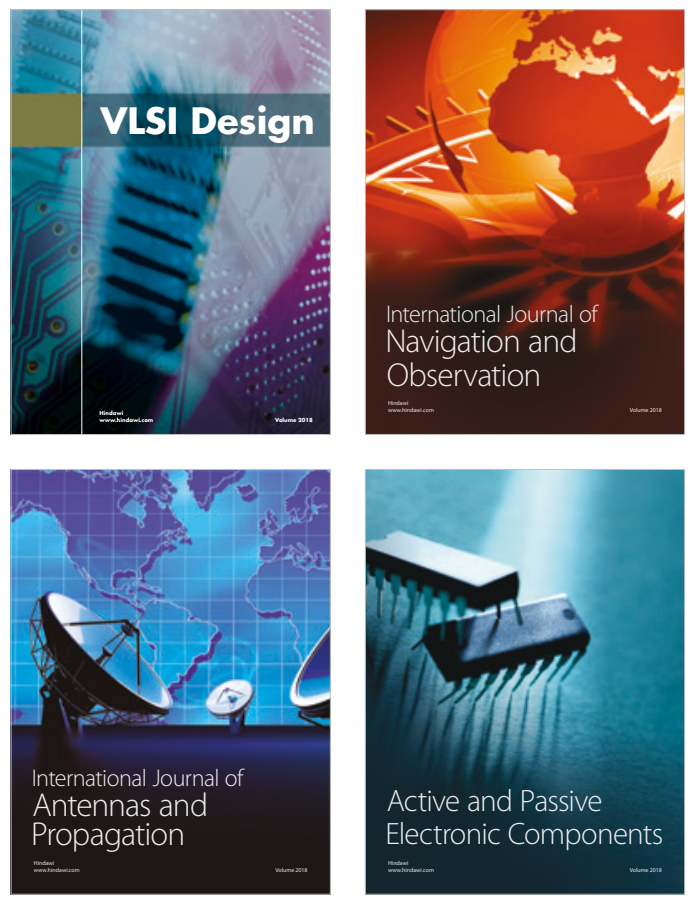
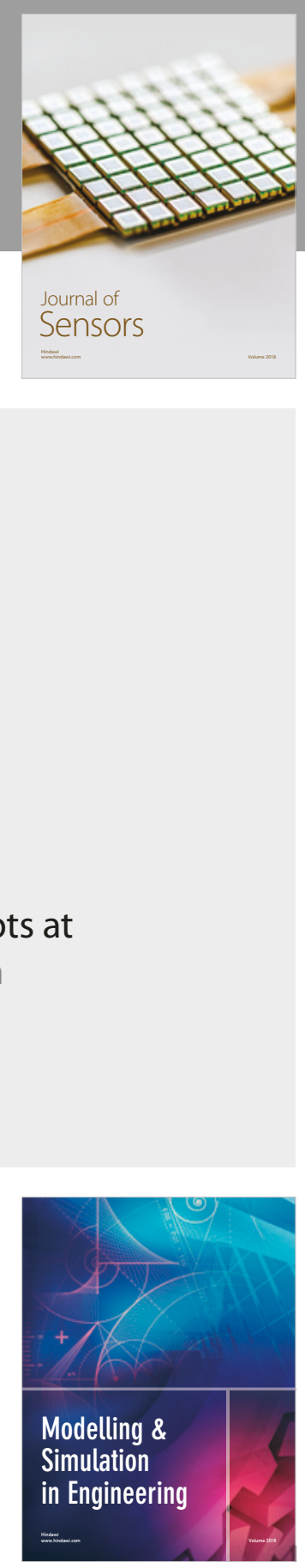

\section{Advances \\ Multimedia}
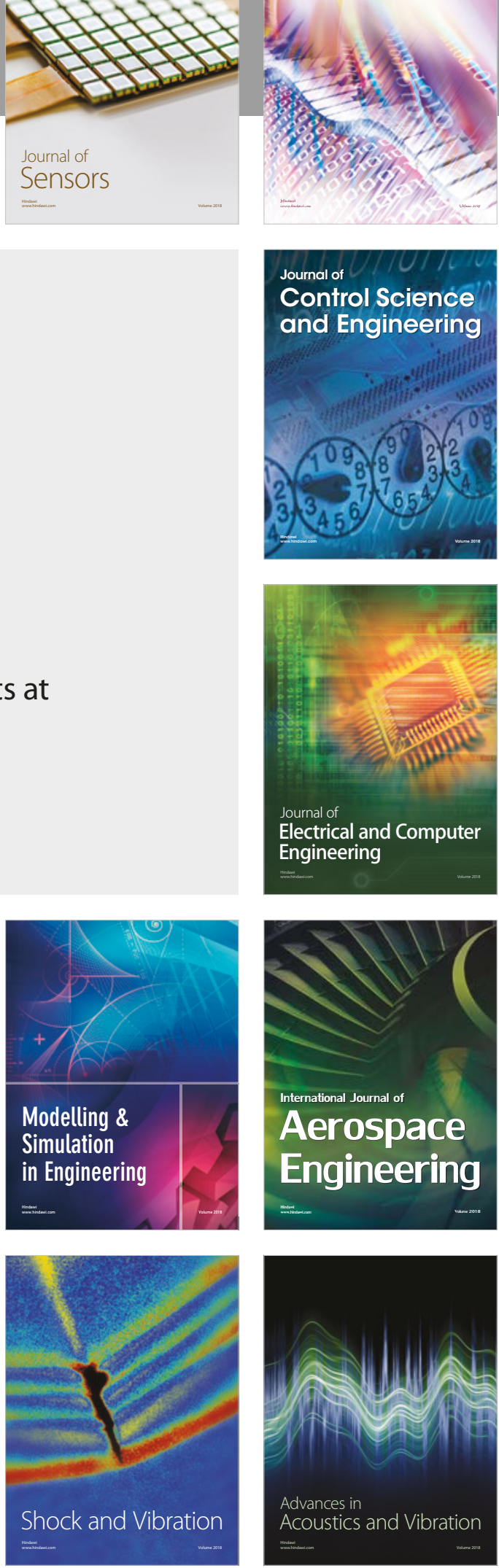